\title{
Global Behavior of a New Rational Nonlinear Higher-Order Difference Equation
}

\author{
Wen-Xiu Ma $\mathbb{D}^{1,2,3,4,5,6}$ \\ ${ }^{1}$ Department of Mathematics, Zhejiang Normal University, Jinhua 321004, Zhejiang, China \\ ${ }^{2}$ Department of Mathematics, King Abdulaziz University, Jeddah, Saudi Arabia \\ ${ }^{3}$ Department of Mathematics and Statistics, University of South Florida, Tampa, FL 33620-5700, USA \\ ${ }^{4}$ College of Mathematics and Physics, Shanghai University of Electric Power, Shanghai 200090, China \\ ${ }^{5}$ College of Mathematics and Systems Science, Shandong University of Science and Technology, Qingdao 266590, Shandong, China \\ ${ }^{6}$ Department of Mathematical Sciences, North-West University, Mafikeng Campus, Mafikeng, Mmabatho 2735, South Africa
}

Correspondence should be addressed to Wen-Xiu Ma; mawx@cas.usf.edu

Received 10 April 2019; Revised 22 June 2019; Accepted 14 July 2019; Published 7 August 2019

Academic Editor: Mondher Farza

Copyright (c) 2019 Wen-Xiu Ma. This is an open access article distributed under the Creative Commons Attribution License, which permits unrestricted use, distribution, and reproduction in any medium, provided the original work is properly cited.

Let $k$ be a nonnegative integer and $c$ a real number greater than or equal to 1 . We present qualitative global behavior of solutions to a rational nonlinear higher-order difference equation $z_{n+1}=\left(c\left(z_{n}+z_{n-k}\right)+(c-1) z_{n} z_{n-k}\right) /\left(z_{n} z_{n-k}+c\right), n \geq 0$, with positive initial values $z_{-k}, z_{-k+1}, \cdots, z_{0}$, and show the global asymptotic stability of its positive equilibrium solution.

\section{Introduction}

Difference equations have wide applications in biology, computer science, digital signal processing, and economics. A general solution structure exists for linear difference equations [1]. However, in various situations of nonlinear higher-order difference equations, solution properties can only be observed by numerical simulation, and it is often exceedingly difficult to give a full mathematical proof for the properties predicted by numerical simulation and the conclusions formed on the basis of guesswork [2]. It is, therefore, important to make qualitative analysis on nonlinear higherorder difference equations, which is the topic of the current study. There have been some related studies on rational nonlinear difference equations in the literature (see, e.g., [38]). Global asymptotic properties of spectral functions are also crucial in determining algebro-geometric solutions of soliton equations (see, e.g., $[9,10]$ ) and scattering data in matrix spectral problems (see, e.g., [11]).

An iterative algorithm to approximate a zero of a given function $f$ reads

$$
x_{n+1}=\frac{x_{n-1} f\left(x_{n}\right)-x_{n} f\left(x_{n-1}\right)}{f\left(x_{n}\right)-f\left(x_{n-1}\right)}, \quad n \geq 0,
$$

and an application of this to a quadratic function $f(x)=x^{2}-$ $a, a>0$, gives

$$
x_{n+1}=\frac{x_{n} x_{n-1}+a}{x_{n}+x_{n-1}}, \quad n \geq 0 .
$$

Let $k$ be a nonnegative integer and $c$ a real number greater than or equal to 1 . We would like to consider a more general rational nonlinear higher-order difference equation

$$
z_{n+1}=\frac{c\left(z_{n}+z_{n-k}\right)+(c-1) z_{n} z_{n-k}}{z_{n} z_{n-k}+c}, \quad n \geq 0,
$$

with positive initial values $z_{-k}, z_{-k+1}, \cdots, z_{0}$, which engender positive solutions. We take a transformation

$$
z_{n}=\frac{c}{y_{n}}, \quad n \geq-k
$$

and then obtain another difference equation

$$
y_{n+1}=\frac{y_{n} y_{n-k}+c}{y_{n}+y_{n-k}+c-1}, \quad n \geq 0,
$$

Obviously, the equilibrium solution of the rational nonlinear difference equation (3), $\bar{z}=c$, becomes the equilibrium solution of the transformed difference equation (5), $\bar{y}=1$. 
If we further take $c=1$, then we obtain the nonlinear difference equation discussed in $[6,7]$ :

$$
y_{n+1}=\frac{y_{n} y_{n-k}+1}{y_{n}+y_{n-k}}, \quad n \geq 0 .
$$

Introducing $x_{n}=\sqrt{a} y_{n}$ into (6) yields

$$
x_{n+1}=\frac{x_{n} x_{n-k}+a}{x_{n}+x_{n-k}}, \quad n \geq 0,
$$

where $a>0$. When $k=1$, this gives the nonlinear difference equation in (2). The equation (7) in the case of $k=2$ was studied in [5] and its closed-form solution was presented in [6]. In the general case of $k$, the asymptotic stability of the positive equilibrium solution $\bar{x}=1$ of the equation (7) was proved in [7].

It is direct to see that the rational nonlinear higher-order difference equation, defined by (3), possesses three equilibria: $\bar{z}=-1,0, c$. In this article, we would like to explore global behavior of solutions to the rational nonlinear higher-order difference equation (3), show the global asymptotic stability of its positive equilibrium solution $\bar{z}=c$, and present two illustrative examples of positive solutions.

\section{Global Behavior}

2.1. Classification of Solutions. First of all, based on the rational difference equation (3), one can have

$$
\begin{gathered}
z_{n+1}-c=\frac{\left(c-z_{n-k}\right)\left(z_{n}-c\right)}{z_{n} z_{n-k}+c}, \quad n \geq 0, \\
z_{n+1}-z_{n}=\frac{z_{n-k}\left(z_{n}+1\right)\left(c-z_{n}\right)}{z_{n} z_{n-k}+c}, \quad n \geq 0, \\
z_{n+1}-z_{n-k}=\frac{z_{n}\left(z_{n-k}+1\right)\left(c-z_{n-k}\right)}{z_{n} z_{n-k}+c}, \quad n \geq 0 .
\end{gathered}
$$

Further from (9) and (10), we can easily derive the following solution properties.

Theorem 1. If $\left\{z_{n}\right\}_{n=-k}^{\infty}$ is a solution to the rational nonlinear difference equation (3), then one has

$$
\begin{array}{ll}
z_{n+1}<z_{n} & \text { if } z_{n}>c, \\
z_{n+1}>z_{n} & \text { if } z_{n}<c, \\
z_{n+1}<z_{n-k} & \text { if } z_{n-k}>c, \\
z_{n+1}>z_{n-k} & \text { if } z_{n-k}<c,
\end{array}
$$

where $n \geq 0$.

If $k=0$, the rational difference equation (3) becomes a first-order difference equation

$$
z_{n+1}=\frac{2 c z_{n}+(c-1) z_{n}^{2}}{z_{n}^{2}+c}, \quad n \geq 0 .
$$

Then for $n \geq 0$, one has $z_{n+1} \leq c$, because $-z_{n}^{2}+2 c z_{n} \leq c^{2}$. For $n \geq 1$, one has $z_{n+1} \geq z_{n}$, since $(c-1) z_{n}+c \geq z_{n}^{2}$, due to $z_{n} \leq c$. Therefore, every solution $z_{n}$ decays to $c$, when $n \longrightarrow \infty$.

Generally, there are three types of solutions to the rational nonlinear higher-order difference equation (3).

Theorem 2. Let $k \geq 1$. If $\left\{z_{n}\right\}_{n=-k}^{\infty}$ is a solution to the rational nonlinear higher-order difference equation (3), and then

(a) it is eventually equal to $c$, more precisely $z_{n}=c, n \geq m$, which occurs when $z_{m}=c$ for some $m \geq 0$;

(b) it is eventually less than $c$, more precisely $z_{n}<z_{n+1}<c$, $n \geq m+k$, which occurs when $z_{m}, z_{m+1}, \cdots, z_{m+k}<c$ for some $m \geq-k$; or

(c) it oscillates about $c$, possessing at most $k$ consecutive increasing terms less than $c$ and at most $k+1$ consecutive decreasing terms greater than $c$.

Proof. Equality (8) and property (11) directly tell that we have three types of solutions to the rational nonlinear higher-order difference equation (3).

The decreasing and increasing characteristics of oscillatory solutions in the third solution situation (c) can be proved as follows.

Suppose that $n_{1}, n_{2} \geq 0$ are two integers satisfying $n_{1}<n_{2}$. We express

$$
\begin{aligned}
z_{n_{2}}-z_{n_{1}}= & \left(z_{n_{2}}-z_{n_{2}-1}\right)+\left(z_{n_{2}-1}-z_{n_{2}-2}\right)+\cdots \\
& +\left(z_{n_{1}+1}-z_{n_{1}}\right)=D
\end{aligned}
$$

where $D$ can be written as

$$
D=\sum_{j=n_{1}}^{n_{2}-1} \frac{z_{j-k}\left(c-z_{j}\right)\left(z_{j}+1\right)}{z_{j} z_{j-k}+c}
$$

by (9).

If $z_{n}>c$ for $n_{1} \leq n \leq n_{2}$, then each term in $D$ is less than zero, and so $z_{n_{2}}<z_{n_{1}}$, due to (14). If $z_{n}<c$ for $n_{1} \leq n \leq n_{2}$, then each term in $D$ is greater than zero, and so $z_{n_{2}}>z_{n_{1}}$, due to (14). This completes the proof.

Note that based on (8), we can see that there is no solution situation that a solution of (3) is eventually greater than $c$.

2.2. Global Asymptotic Stability. When $k=0$, the equilibrium solution $\bar{z}=c$ of the first-order rational difference equation (13) is globally asymptotically stable, since it is a globally attractive equilibrium solution of a first-order difference equation (see [12] for a general theory).

For a general $k \geq 1$, we can show the same global asymptotic stability of the positive equilibrium solution $\bar{z}=c$ of the rational nonlinear difference equation (3), by establishing the local asymptotic stability and the global attractivity, which imply the global asymptotic stability [2]. Instead, we establish a strong negative feedback property [13] to guarantee the global asymptotic stability of $\bar{z}=c$ (see [14] for details on the strong negative feedback property). 
Theorem 3. The positive equilibrium solution $\bar{z}=c$ of the rational nonlinear higher-order difference equation (3) is globally asymptotically stable.

Proof. Based on the rational nonlinear difference equation (3), one can have

$$
\begin{aligned}
& \frac{c^{2}}{z_{n-k}}-z_{n+1} \\
& =\frac{\left(c-z_{n-k}\right)\left[(c-1) z_{n} z_{n-k}+c z_{n-k}+c^{2}\right]}{z_{n-k}\left(z_{n} z_{n-k}+c\right)},
\end{aligned}
$$

$$
n \geq 0 \text {. }
$$

From this equality and the equality in (10), we can obtain

$$
\begin{array}{r}
\left(z_{n-k}-z_{n+1}\right)\left(\frac{c^{2}}{z_{n-k}}-z_{n+1}\right) \\
=-\frac{z_{n}\left(z_{n-k}+1\right)\left(c-z_{n-k}\right)^{2}\left[(c-1) z_{n} z_{n-k}+c z_{n-k}+c^{2}\right]}{z_{n-k}\left(z_{n} z_{n-k}+c\right)^{2}}, \\
\quad n \geq 0,
\end{array}
$$

which leads to a strong negative feedback property:

$$
\left(z_{n-k}-z_{n+1}\right)\left(\frac{c^{2}}{z_{n-k}}-z_{n+1}\right) \leq 0, \quad n \geq 0
$$

with equality for all $n \geq 0$ if and only if $z_{n}=c, n \geq-k$. It, therefore, follows from a stability theorem (Corollary 3 of [14]) that the equilibrium solution $\bar{z}=c$ of the rational nonlinear difference equation (3) is globally asymptotically stable. Thus, the proof is finished.

The above theorems with $c=1$ gives the results in [4] $(k=1),[5](k=2)$ and [7] (a general $k$ ). There have also been similar studies on polynomial difference equations (see, for example, [15]) and other studies on positive rational function solutions, called lumps, to partial differential equations (see, e.g., [16]).

2.3. Illustrative Examples and an Open Question. To illustrate the global properties stated in Theorems 2 and 3, here we present two illustrative examples associated with two special cases:

$$
\begin{aligned}
c & =\frac{3}{2}, \\
k & =3, \\
z_{-3} & =\frac{6}{5}, \\
z_{-2} & =\frac{16}{9}, \\
z_{-1} & =\frac{10}{7},
\end{aligned}
$$

$$
\begin{aligned}
z_{0} & =1 ; \\
c & =2, \\
k & =5, \\
z_{-5} & =\frac{5}{4}, \\
z_{-4} & =\frac{7}{3}, \\
z_{-3} & =\frac{5}{3}, \\
z_{-2} & =\frac{9}{4} \\
z_{-1} & =\frac{3}{2}, \\
z_{0} & =\frac{7}{3} ;
\end{aligned}
$$

in Figure 1. From the plot pictures, we see that the convergence is achieved very fast in both cases.

Finally, let $k \geq 1$. For an oscillatory solution $\left\{z_{n}\right\}_{n=-k}^{\infty}$ of the rational nonlinear difference equation (3), we define

$$
\begin{aligned}
N_{g} & =\left\{n \mid z_{n}>c \text { and } n \geq 0\right\}, \\
N_{l} & =\left\{n \mid z_{n}<c \text { and } n \geq 0\right\} .
\end{aligned}
$$

Since $\left\{z_{n}\right\}_{n=-k}^{\infty}$ is oscillatory, Theorem 2 guarantees that both $N_{g}$ and $N_{l}$ have infinitely many numbers. A basic open question that we are very interested in is if $z_{n}$ is decreasing on $N_{g}$ and increasing on $N_{l}$. We point out that through the above two examples, we failed to find any counterexample to this statement, but found that two cases could occur: either $z_{n-1}, z_{n+1}<c$ but $z_{n}>c$ or $z_{n-1}, z_{n+1}>c$ but $z_{n}<c$ for some $n>1$.

\section{Data Availability}

The data used to support the findings of this study are included within the article.

\section{Conflicts of Interest}

The authors declare that there are no conflicts of interest regarding the publication of this paper.

\section{Acknowledgments}

This study was in part supported by NNSFC under Grants 11371326, 11371086, and 11571079, NSF under Grant DMS1664561, and the distinguished professorships of King Abdulaziz University, Saudi Arabia and North-West University, South Africa. 

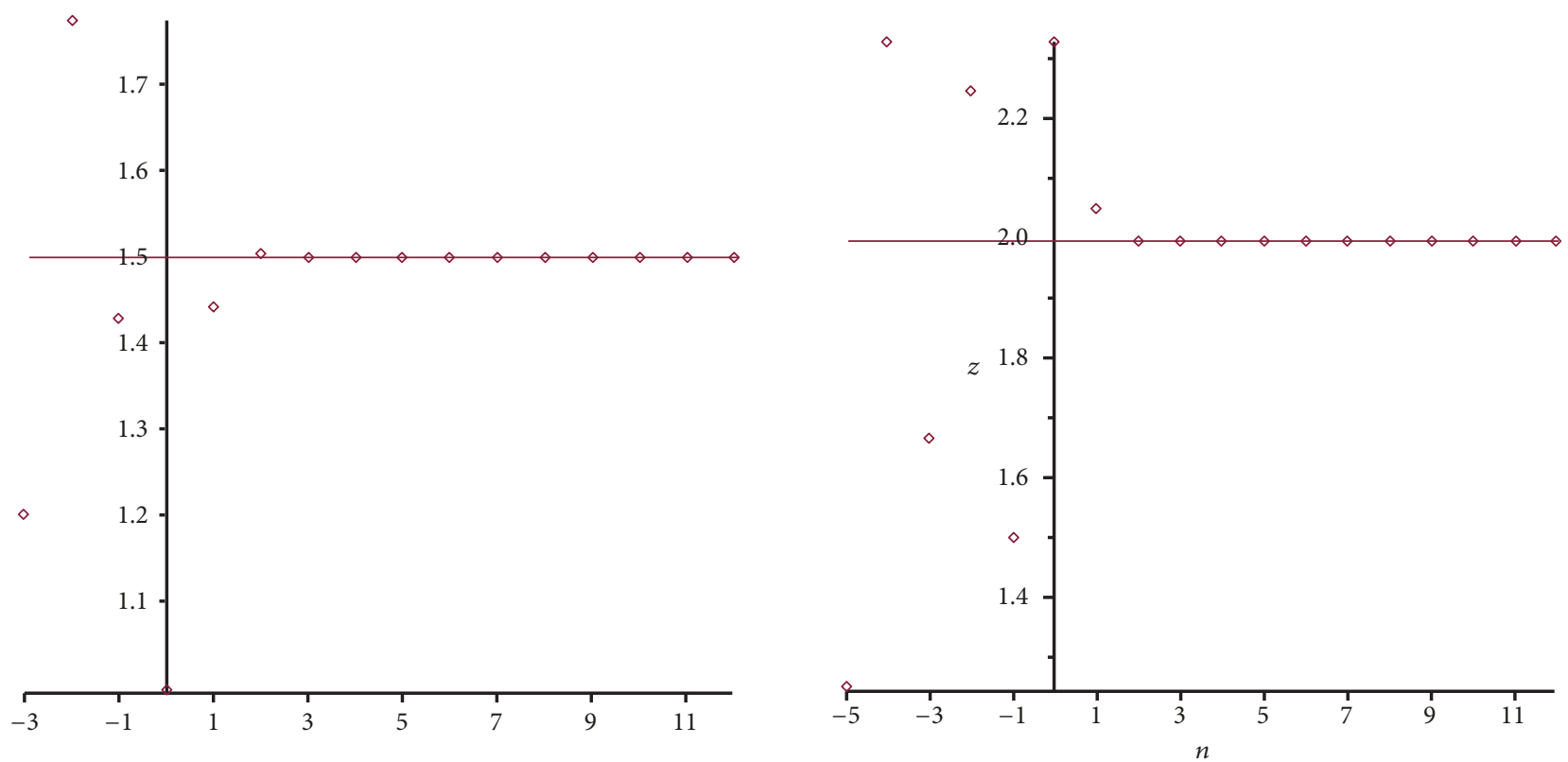

Figure 1: Profiles of $\left\{z_{n}\right\}_{n=-k}^{\infty}$ with $c=3 / 2, k=3$ (left) and $c=2, k=5$ (right).

\section{References}

[1] P. M. Batchelder, An Introduction to Linear Difference Equations, Dover Publications, New York, NY, USA, 1967.

[2] V. L. Kocic and G. Ladas, Global behavior of Nonlinear Difference Equations of Higher Order with Applications, Kluwer, Dordrecht, The Netherlands, 1993.

[3] E. Camouzis, G. Ladas, I. W. Rodrigues, and S. Northshield, "The rational recursive sequence $x_{n+1}=\beta x_{n}^{2} /\left(1+x_{n-1}^{2}\right)$," Computers and Mathematics with Applications, vol. 28, no. 1-3, pp. 37-43, 1994.

[4] X. Li and D. Zhu, "Global asymptotic stability in a rational equation," Journal of Difference Equations and Applications, vol. 9, no. 9, pp. 833-839, 2003.

[5] X. Li and D. Zhu, “Two rational recursive sequences," Computers \& Mathematics with Applications, vol. 47, no. 10-11, pp. 14871494, 2004.

[6] M. Ben H. Rhouma, "The Fibonacci sequence modulo $\pi$, chaos and some rational recursive equations," Journal of Mathematical Analysis and Applications, vol. 310, no. 2, pp. 506-517, 2005.

[7] R. Abu-Saris, C. Çina, and I. Yalçinkaya, "On the asymptotic stability of $x_{n+1}=\left(a+x_{n} x_{n-k}\right) /\left(x_{n}+x_{n-k}\right)$," Computers \& Mathematics with Applications, vol. 56, no. 5, pp. 1172-1175, 2008.

[8] A. Gelişken, C. Çinar, and A. S. Kurbanli, "On the asymptotic behavior and periodic nature of a difference equation with maximum," Computers \& Mathematics with Applications, vol. 59, no. 2, pp. 898-902, 2010.

[9] W. X. Ma, "Trigonal curves and algebro-geometric solutions to soliton hierarchies I," Proceedings of the Royal Society A: Mathematical, Physical and Engineering Sciences, vol. 473, no. 2203, p. 20170232, 2017.

[10] W. X. Ma, “Trigonal curves and algebro-geometric solutions to soliton hierarchies II," Proceedings of the Royal Society A: Mathematical, Physical and Engineering Sciences, vol. 473, no. 2203, p. 20170233, 2017.
[11] W. X. Ma, "The inverse scattering transform and soliton solutions of a combined modified Korteweg-de Vries equation," Journal of Mathematical Analysis and Applications, vol. 471, no. 1-2, pp. 796-811, 2019.

[12] H. Sedaghat, "The impossibility of unstable, globally attracting fixed points for continuous mappings of the line," The American Mathematical Monthly, vol. 104, no. 4, pp. 356-358, 1997.

[13] A. Amleh, N. Kruse, and G. Ladas, "On a class of difference equations with strong negative feedback," Journal of Difference Equations and Applications, vol. 5, no. 6, pp. 497-515, 1999.

[14] N. Kruse and T. Nesemann, "Global asymptotic stability in some discrete dynamical systems," Journal of Mathematical Analysis and Applications, vol. 235, no. 1, pp. 151-158, 1999.

[15] X. Li and D. Zhu, "Global asymptotic stability for two recursive difference equations," Applied Mathematics and Computation, vol. 150, no. 2, pp. 481-492, 2004.

[16] W. X. Ma and Y. Zhou, "Lump solutions to nonlinear partial differential equations via Hirota bilinear forms," Journal of Differential Equations, vol. 264, no. 4, pp. 2633-2659, 2018. 


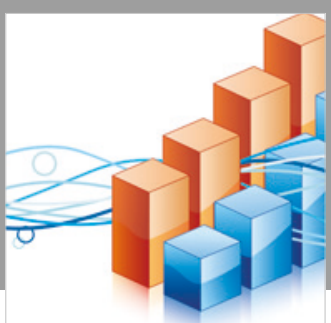

Advances in

Operations Research

\section{-n-m}
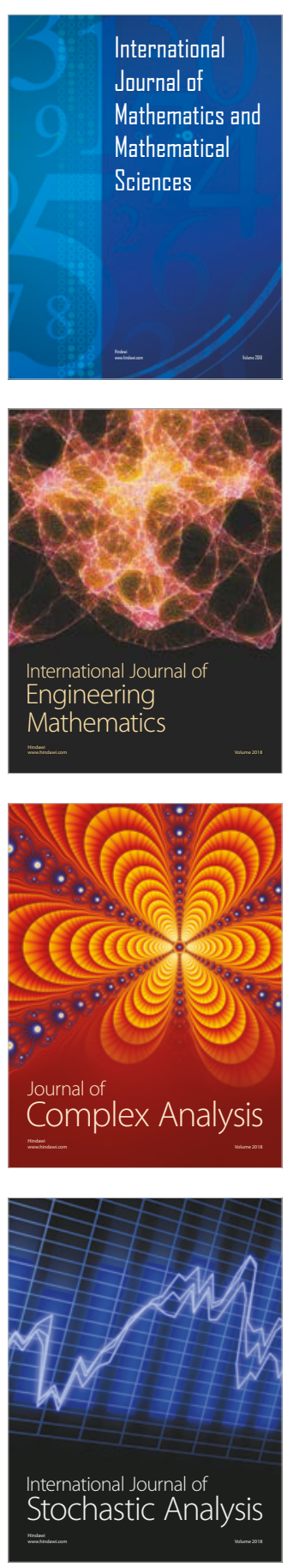
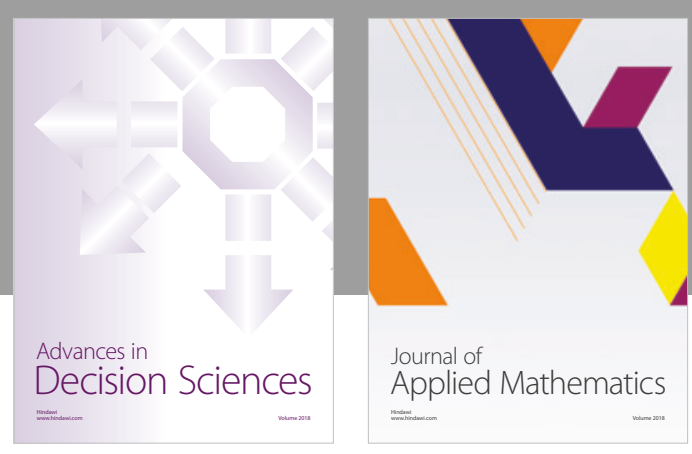

Journal of

Applied Mathematics
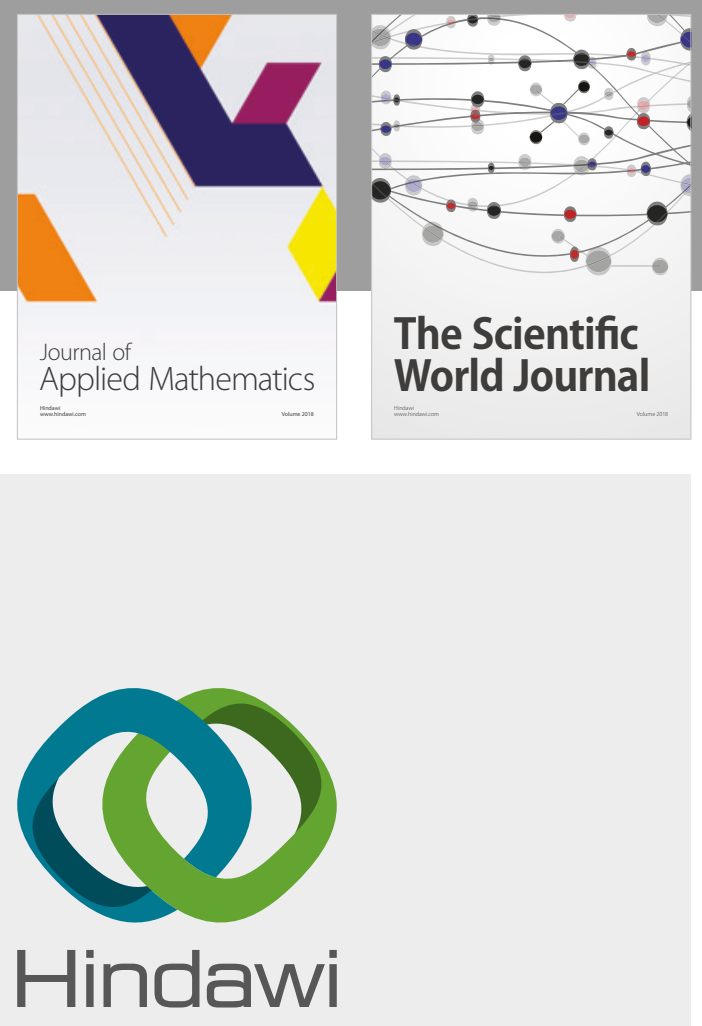

Submit your manuscripts at

www.hindawi.com

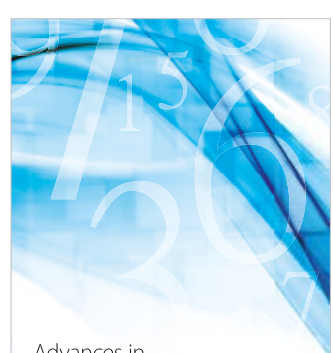

Advances in
Numerical Analysis
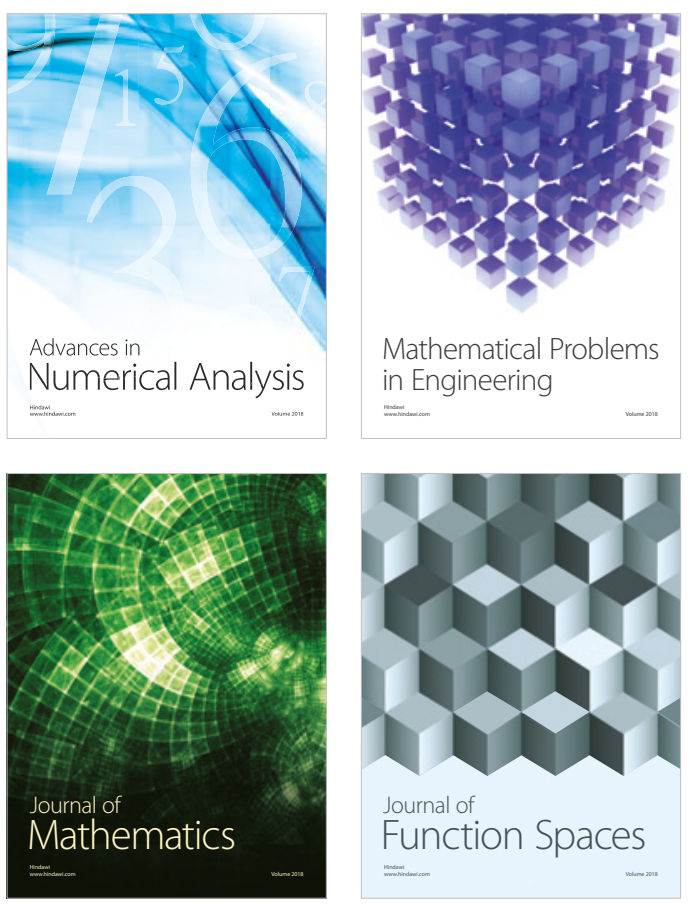

Mathematical Problems in Engineering

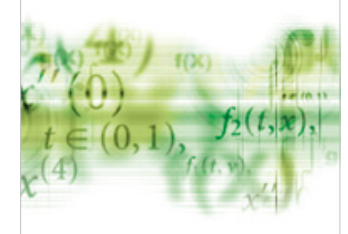

International Journal of

Differential Equations

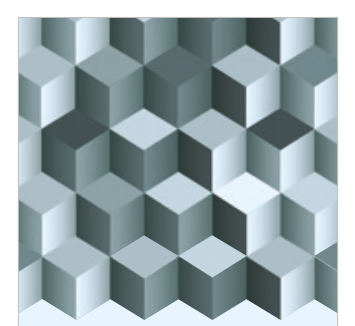

Journal of

Function Spaces
The Scientific

World Journal

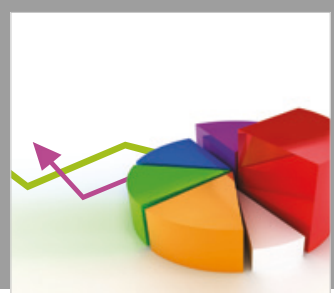

Journal of

Probability and Statistics
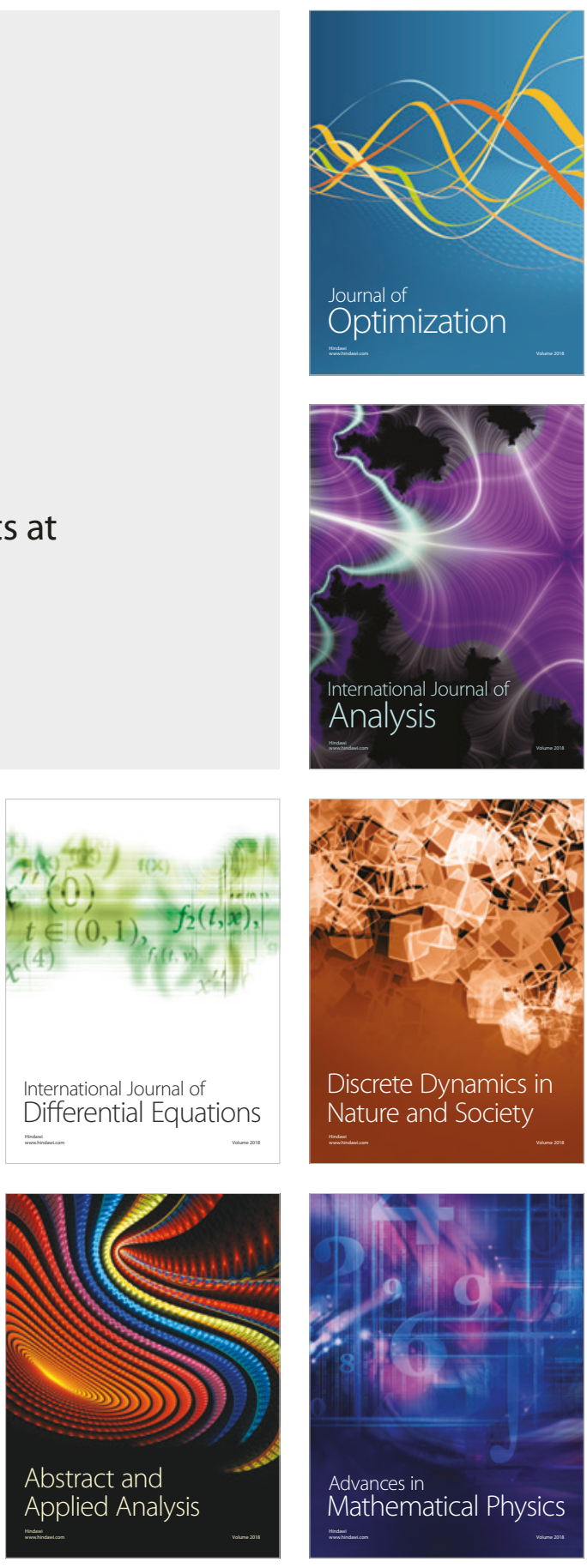